\title{
The Incidence of Complex Regional Pain Syndrome After Fasciectomy for Dupuytren's Contracture: A Prospective Observational Study of Four Anesthetic Techniques
}

\author{
Scott S. Reuben, MD, Rene Pristas, MD, Duane Dixon, MD, Shameema Faruqi, MD, \\ Lakshmi Madabhushi, MD, and Steven Wenner, MD \\ Departments of Anesthesiology and Orthopedic Surgery, Baystate Medical Center, Springfield, Massachusetts; \\ Tufts University School of Medicine, Boston, Massachusetts
}

\begin{abstract}
The development of complex regional pain syndrome (CRPS) is not an uncommon complication after Dupuytren's surgery. Despite increasing research interest, little is known regarding which patients are at increased risk for developing CRPS and what is the optimal perioperative treatment strategy for preventing the occurrence of this disease after surgery. We prospectively evaluated the use of four anesthetic techniques (general anesthesia, axillary block, and IV regional anesthesia [IVRA] with lidocaine with or without clonidine) for patients undergoing fasciectomy for Dupuytren's contracture. All patients were followed in the Pain Management Center at 1,3, and
\end{abstract}

12 mo postoperatively by a blinded physician to evaluate the presence of CRPS. Significantly $(P<0.01)$ more patients developed postoperative CRPS in the general anesthesia group ( $n=25 ; 24 \%$ ) and the IVRA lidocaine group ( $n=12 ; 25 \%$ ) compared with either the axillary block group $(n=5 ; 5 \%)$ or the IVRA lidocaine and clonidine group $(n=3 ; 6 \%)$. We conclude that axillary block or IVRA with clonidine offers a significant advantage for decreasing the incidence of CRPS compared with either IVRA with lidocaine alone or general anesthesia for patients undergoing Dupuytren's surgery.

(Anesth Analg 2006;102:499-503)
C omplex regional pain syndrome (CRPS), previously known as "reflex sympathetic dystrophy," is used to describe a syndrome of pain, sudomotor, and/or vasomotor instability (1). This pain usually has an initiating noxious event in the periphery, is not limited to the distribution of a single nerve, and is disproportionate to the inciting event (1-3). The Consensus Conference of the International Association for the Study of Pain (IASP) has subdivided CRPS into two forms: CRPS type I (formerly reflex sympathetic dystrophy) and CRPS II (formerly causalgia) (4). According to the IASP, the diagnosis of CRPS I requires 1) continuing pain, allodynia, or hyperalgesia disproportionate to the injury; 2) evidence at some time of edema, changes in skin blood flow, or abnormal sudomotor activity in the region of pain; and 3) no

Presented, in part, at the Annual American Society of Anesthesiologists Meeting, Las Vegas, NV 10/04.

Support was provided solely from institutional and/or departmental sources.

Accepted for publication September 23, 2005.

Address correspondence and reprint requests to Scott S. Reuben, MD, Department of Anesthesiology, Baystate Medical Center, 759 Chestnut Street, Springfield, MA 01199, (413) 794-3520, (413) 7945349 FAX; email: scott.reuben@bhs.org.

DOI: 10.1213/01.ane.0000194879.85643.ff other conditions that would otherwise account for the degree of pain and dysfunction (2). Motor disturbances and trophic changes, such as altered nail and hair growth, may be observed in some cases. CRPS II is a pain syndrome that starts after a nerve injury and is not necessarily limited to the distribution of the injured nerve (5). The diagnostic criteria are the same as those of CRPS I. Patients with CRPS I or CRPS II can have sympathetically maintained pain or sympathetically independent pain. "Sympathetically maintained pain," a term introduced in 1986 by Roberts (6), is pathologic pain that is supported by sympathetic efferent activity, circulating catecholamines, and/or increased sensitivity of $\alpha$-adrenergic receptors. Sympathetically maintained pain is identified by the ability to lessen the pain by sympatholytic blocks or interventions. Sympathetically independent pain has components of pain from sources other than sympathetic innervation and is believed to be most commonly observed in advanced cases of CRPS that do not respond to sympathetic blocks (6). Patients with CRPS may present with components only of sympathetically maintained pain or sympathetically independent pain, or, more commonly, a combination of pain from each (7). 
Despite increasing research interest, little is known regarding which patients are at increased risk for developing CRPS or what might be the optimal perioperative treatment strategy for preventing the occurrence of this disease after surgery $(8,9)$. The development of CRPS is not an uncommon complication after orthopedic surgery. CRPS after fasciectomy for Dupuytren's contracture has been reported to occur in $4.5 \%$ to $40 \%$ of patients (10-14).

It has been reported that regional anesthesia, by allowing the preoperative onset of sympathetic blockade, may be a more appropriate anesthetic choice for preventing the development of postoperative CRPS $(15,16)$. Further, it has been proposed that sympathetically maintained pain may be rekindled by surgery performed under general anesthesia (17). However, no previous study has validated the appropriate anesthetic technique for preventing CRPS. Currently, we perform Dupuytren's surgery using either general anesthesia, axillary brachial plexus block, or IV regional anesthesia (IVRA) with or without clonidine. The goal of this prospective study was to assess the incidence of postoperative CRPS using one of these four anesthetic techniques for surgical correction of Dupuytren's contracture.

\section{Methods}

Between 2001 and 2004, 300 consecutive ASA physical status I-III patients undergoing fasciectomy for $\mathrm{Du}-$ puytren's contracture gave written informed consent for this prospective study, which was approved by our IRB. All patients participating in this investigation had not participated in other CRPS studies at our institution and there was no cross-over of patients from one study to another. All procedures were performed with the use of a pneumatic tourniquet under general anesthesia $(n=106)$, axillary brachial plexus block $(n=96)$, IVRA with lidocaine (IVRA-L) $(n=48)$, or IVRA with lidocaine and clonidine (IVRA-C) $(n=$ 50). The anesthetic technique used was based on surgeon, anesthesiologist, and/or patient preference. Patients receiving general anesthesia were administered propofol $\left(2 \mathrm{mg} / \mathrm{kg}\right.$ ) for induction and $30 \%-50 \% \mathrm{~N}_{2} \mathrm{O}$ in $\mathrm{O}_{2}$, fentanyl $(2-3 \mu \mathrm{g} / \mathrm{kg})$, and sevoflurane $1 \%-2 \%$ end-tidal concentration delivered via laryngeal mask airway. For brachial plexus anesthesia, axillary blockade was performed with the patient in the supine position and the upper arm abducted $90^{\circ}$ and the elbow flexed at $110^{\circ}$. The axillary artery was palpated and a 22-gauge 5-cm Stimuplex needle (B Braun, Bethlehem, PA) connected to a nerve stimulator was inserted into the proximal part of the axilla. The needle was considered to be placed correctly when contraction of the biceps or muscle groups in the forearm was seen in response to stimuli of $\leq 0.5 \mathrm{~mA}$. All patients received a $40 \mathrm{~mL}$ injection containing $20 \mathrm{~mL} \mathrm{2.0 \%}$
Table 1. Diagnostic Criteria for Complex Regional Pain Syndrome

1. Spontaneous or evoked pain out of proportion to the surgical procedure

2. At least 4 of the following signs or symptoms:

- Sensory changes, including allodynia and hyperesthesia

- Vasomotor changes

- Sudomotor changes

- Temperature difference $\left(\geq 1^{\circ} \mathrm{C}\right)$ between extremities

- Diffuse edema

- Limited active range of motion

3. Occurrence or increase of above signs or symptoms after use of the arm

4. Above signs or symptoms present in an area much larger than the area of operation

lidocaine with epinephrine $5 \mu \mathrm{g} / \mathrm{mL}$ and $20 \mathrm{~mL} \mathrm{0.5 \%}$ bupivacaine. For patients receiving IVRA, a $14-\mathrm{cm}$ double tourniquet positioned on the upper operative arm was used. The operative extremity was exsanguinated by elevating and wrapping it with a $10-\mathrm{cm}$ Esmarch bandage. The proximal tourniquet was inflated to $100 \mathrm{~mm} \mathrm{Hg}$ more than the systolic blood pressure to a minimum of $250 \mathrm{~mm} \mathrm{Hg}$, and the Esmarch bandage was removed. Circulatory isolation of the operative arm was confirmed by inspection of the hand and by absence of the radial pulse. IVRA was established using $40 \mathrm{~mL} 0.5 \%$ lidocaine with or without clonidine $1 \mu \mathrm{g} / \mathrm{kg}$ administered over 2-3 min.

All patients were followed in the Pain Management Center at 1,3, and 12 mo postoperatively by a blinded physician to determine the presence of CRPS. The diagnosis of CRPS was based on the IASP criteria (2), as summarized in Table 1. All patients presented with spontaneous or evoked pain out of proportion to the surgical procedure. In addition, at least 4 of the 6 following signs or symptoms were present: sensory changes including allodynia and hyperesthesia, vasomotor changes, sudomotor changes, temperature difference $\left(\geq 1^{\circ} \mathrm{C}\right)$ between upper extremities, diffuse edema, and limited active range of motion. Cutaneous temperature was measured on both the first digit of the affected and contralateral extremity using a YSI $4000 \mathrm{~A}$ temperature probe (Yellow Springs Instrument Corporation, Yellow Springs, $\mathrm{OH})$. These symptoms should increase in severity with use of the affected extremity. In addition, these symptoms should be present in an area that is larger than the area of the operation.

All data were entered into an Microsoft Excel (Redmond, WA) database and converted to a SAS file (SAS Institute Inc., Cary, NC) for statistical analysis. Demographic data, surgical procedures, duration of surgery, and tourniquet time were analyzed by analysis of variance. The incidence of CRPS was evaluated with the $\chi^{2}$ test. Bonferroni corrections for multiple comparisons 
Table 2. Patient Demographics and Surgical Data

\begin{tabular}{lcccc}
\hline & General Anesthesia & Axillary Block & IVRA Lidocaine & IVRA Clonidine \\
\hline Number & 106 & 96 & 48 & 50 \\
Gender (M/F) & $74 / 32$ & $70 / 26$ & $35 / 13$ & $38 / 12$ \\
Age (yr) & $61 \pm 12$ & $63 \pm 19$ & $65 \pm 16$ & $66 \pm 17$ \\
Weight (kg) & $79 \pm 15$ & $75 \pm 19$ & $81 \pm 18$ & $78 \pm 16$ \\
Duration of surgery (min) & $75 \pm 17$ & $72 \pm 15$ & $89 \pm 16$ & $70 \pm 18$ \\
Tourniquet time (min) & $80 \pm 16$ & $83 \pm 14$ & $81 \pm 15$ & $79 \pm 16$ \\
\hline
\end{tabular}

Data are presented as mean $\pm \mathrm{SD}$. There were no statistical differences among the four groups. IVRA = intravenous regional anesthesia.

were used where applicable. Differences were considered significant at $P<0.05$. For the frequency of CRPS, a sample size of 40 patients for each group was determined by a power analysis based on the following assumptions: 1) $40 \%$ of the patients would experience CRPS under general anesthesia $(8,13) ; 2)$ a decrease of $20 \%$ (e.g., from $40 \%$ to $20 \%$ ) would be considered of clinical importance; and 3) $\alpha=0.05$ and $\beta=0.20$.

\section{Results}

There were no differences in demographic variables, procedures, or tourniquet times among the four groups (Table 2). Four patients in the IVRA-L group required conversion to general anesthesia because of intolerable tourniquet pain. The results of these four patients are included in the data analysis for the IVRA-L group. Five patients developed postoperative complications, including four hematomas and one infection. Two patients who developed a hematoma received IVRA-C, one patient received general anesthesia, and one patient had an axillary block. The only patient who developed a postoperative infection received general anesthesia. All five of these patients later developed CRPS. Significantly $(P<0.01)$ more patients developed postoperative CRPS in the general anesthesia group $(n=25 ; 24 \%)$ and IVRA-L group $(n=12 ; 25 \%)$ compared with either the axillary block group $(n=5 ; 5 \%)$ or the IVRA-C group $(n=3 ; 6 \%)$.

\section{Discussion}

The development of CRPS after fasciectomy for treatment of Dupuytren's disease is a common complication (10-14). Although it has been reported that regional anesthesia may be the most effective technique for preventing the occurrence of CRPS after orthopedic surgery $(15,16)$ and that general anesthesia should be avoided (17), no previous study has validated this practice. Our work clearly demonstrates an advantage to performing either axillary blockade or IVRA with clonidine for patients undergoing Dupuytren's surgery. There was at least a fourfold reduction in the incidence of CRPS with the use of either of these two regional anesthetic techniques. In contrast, the use of
IVRA with lidocaine alone provided no beneficial effect in reducing the incidence of CRPS compared with general anesthesia.

Although IVRA is considered a "regional anesthetic" technique, it does not provide for a perioperative sympathectomy and, therefore, may not prevent the development of CRPS. For those patients undergoing Dupuytren's surgery under IVRA it may be beneficial to perform a perioperative stellate ganglion block or add clonidine to the local anesthetic solution. We have previously demonstrated that using a perioperative stellate ganglion block in patients undergoing upper extremity surgery under IVRA and with a history of CRPS can significantly reduce the incidence of this disease (18). In that study, 100 CRPS patients undergoing surgery on the affected extremity either received a stellate ganglion block $(n=50)$ or no intervention $(n=50)$. The recurrence of CRPS was significantly less in those patients receiving a perioperative stellate ganglion block $(n=5 ; 10 \%)$ compared with those receiving no intervention $(n=36 ; 72 \%)$. No study has examined the efficacy of administering a stellate ganglion block to patients undergoing Dupuytren's surgery without a history of CRPS.

Although the regional sympatholysis provided by a stellate ganglion block may be beneficial in reducing CRPS, it requires clinical expertise and may result in significant morbidity, including vertebral artery injection, subarachnoid, or epidural block, and pneumothorax (19). Further, stellate ganglion blocks frequently do not produce complete sympathetic interruption of the ipsilateral upper extremity (20). We believe IV regional blocks with clonidine may offer an advantage in the perioperative management of patients undergoing fasciectomy for Dupuytren's contracture. Prospective, randomized, controlled clinical trials have examined the efficacy of IV regional blocks with guanethidine, reserpine, droperidol, atropine, bretyllium, and ketanserin in the management of CRPS (21-23). Critical reviews (21-23) of these controlled clinical trials have suggested that there was limited support of analgesic effectiveness of IV regional blocks with bretyllium and ketanserin, consistent data indicating guanethidine and reserpine IV regional blocks were ineffective, and limited data indicating that droperidol and atropine IV regional 
blocks were ineffective. We have previously shown that IVRA with lidocaine and the $\alpha_{2}$-adrenergic agonist, clonidine $(1 \mu \mathrm{g} / \mathrm{kg})$, is an effective technique for managing both acute postoperative pain (24) and symptoms of CRPS (25). Based on these studies $(24,25)$, we have found the complications of IVRA with clonidine are infrequent, and this technique is technically easier to perform than a stellate ganglion block. We recently evaluated the effectiveness of IVRA with lidocaine and clonidine in preventing the recurrence of CRPS after hand surgery (26). In this prospective, randomized, double-blind study, 84 patients with a history of CRPS received either IVRA-L or IVRA-C $(1 \mu \mathrm{g} / \mathrm{kg})$ for anesthesia during hand surgery. The recurrence rate of CRPS was significantly less in those patients receiving IVRA-C (10\%) compared with those patients receiving IVRA-L $(74 \%)$. Clonidine has peripheral analgesic properties in patients with sympathetically maintained pain, possibly because it reduces the release of norepinephrine from prejunctional $\alpha_{2}$ adrenoceptors in the periphery (27). Data from several clinical investigations support the importance of peripheral adrenergic receptors in the maintenance of sympathetically maintained pain. First, $\alpha$-adrenergic blockade with IV administered phentolamine (28), phenoxybenzamine (29), or prazosin (30) reduces pain. Second, IVRA with guanethidine depletes peripheral catecholamines and can relieve sympathetically maintained pain (31). Third, intradermal injection of norepinephrine rekindles sympathetically maintained pain in patients who have previously undergone a sympathectomy (32). Fourth, topical application of clonidine has been shown to eliminate hyperalgesia at the site of drug application only. This hyperalgesia was later rekindled by the intradermal injection of norepinephrine or phenylephrine (33).

IV regional blocks with guanethidine have been studied as a method of decreasing the postoperative incidence of CRPS after Dupuytren's surgery (34). IV regional blocks with guanethidine, which deplete norepinephrine in post-ganglionic adrenergic nerves, were first described in 1974 by Hannington-Kiff (35) as a potential treatment modality for CRPS patients. Sennwald (13) later advocated the perioperative prophylactic use of IV regional blocks with guanethidine in female patients undergoing fasciectomy for $\mathrm{Du}$ puytren's disease because he observed a 40\% incidence of CRPS in this surgical population. However, this practice could not be validated in a prospective, randomized, double-blind study of 71 patients undergoing fasciectomy (34). Patients were randomized to receive either IV regional blocks containing $20 \mathrm{mg}$ of guanethidine or placebo. This study revealed that 7 patients developed CRPS; 5 in the guanethidine group and 2 in the placebo group. The authors concluded that IV regional blocks with guanethidine were an ineffective modality in the prevention of CRPS. These findings are consistent with the other data showing a lack of efficacy for IV regional blocks with guanethidine in the management of CRPS (21-23). However, because of the infrequent incidence of postoperative CRPS $(10 \%)$ observed in this IV regional block guanethidine study (34), it may have been insufficiently powered to demonstrate significant differences between the two treatment groups. Many more patients would have to have been enrolled in this clinical trial before statements pertaining to analgesic efficacy could be made. The infrequent incidence of CRPS may have resulted from the use of brachial plexus block as the primary anesthetic technique $(90 \%)$ in this study. We have shown in the present study, as has been suggested by other investigators (15), that brachial plexus block may reduce the incidence of postoperative CRPS. This reduction in CRPS with axillary blockade may be attributable to the preoperative onset of sympathetic blockade or prolonged postoperative pain relief. Both of these factors play an integral role in the development of postoperative CRPS (8).

Axillary block may be a suitable alternative anesthetic technique for those patients with Dupuytren's contracture in whom IVRA is either contraindicated or cannot be tolerated. Tourniquet pain is often a limiting factor in the use of IVRA and its onset can vary according to the clinical situation. Clinical investigations involving unmedicated volunteers have shown that upper extremity tourniquet inflation can be tolerated for only $29-47$ minutes (36-38). The majority of cases in the present study exceeded this time interval, necessitating tourniquet rotation and/or intraoperative administration of supplemental opioids. In addition, 4 of $48(8.3 \%)$ patients in the IVRA-L group required conversion to general anesthesia because of unbearable tourniquet pain. Although not specifically investigated in the present study, we have previously observed that many patients are able to better tolerate tourniquet pain under axillary block compared with IVRA.

Interestingly, all 5 patients who developed postoperative complications (hematoma or infection) were later diagnosed with CRPS. Although acute surgical pain declines rapidly over the first few days postoperatively, both infection and hematoma have the capacity to produce persistent pain states (39). This continuing postoperative pain may have predisposed patients to more frequent CRPS. It has been hypothesized that one of the pathophysiological mechanisms of CRPS is a continuing barrage of nociceptive input from the peripheral to the central nervous system leading to a state of central hyperexcitability $(40-42)$. Therefore, in addition to performing Dupuytren's surgery under axillary block or IVRA with clonidine, careful attention to improving postoperative pain control and avoiding surgical complications of the affected extremity may play 
an important role in reducing the incidence of postoperative CRPS (8).

In conclusion, axillary block or IVRA with clonidine offers a significant advantage for decreasing the incidence of CRPS compared with either IVRA-L or general anesthesia for patients undergoing Dupuytren's surgery. Because of improved tolerance to tourniquet pain, axillary block may be considered the regional anesthetic technique of choice for this surgical procedure.

\section{References}

1. Stanton-Hicks M, Jänig W, Hassenbusch S, et al. Reflex sympathetic dystrophy: Changing concepts and taxonomy. Pain 1995; 63:127-33.

2. Boas RA. Complex regional pain syndromes: Symptoms, signs, and differential diagnosis. In: Stanton-Hicks, M, Jänig W, eds. Reflex sympathetic dystrophy: A reappraisal. Seattle: IASP Press, 1997:79-92.

3. Raja SN, Grabow TS. Complex regional pain syndrome I (reflex sympathetic dystrophy). Anesthesiology 2002;96:1254-60.

4. Merskey KR, Bogduk N. Classification of chronic pain: Description of chronic pain syndromes and definitions of pain terms, 2nd ed. Seattle: IASP Press, 1994:40-3.

5. Baron R. Peripheral neuropathic pain: from mechanisms to symptoms. Clin J Pain 2000;16:S12-20.

6. Roberts WJ. A hypothesis on the physiologic basis for causalgia and related pains. Pain 1986;24:297-311.

7. Boas RA. Sympathetic nerve blocks: in search of a role. Reg Anesth Pain Med 1998;23:292-305.

8. Reuben SS. Preventing the development of complex regional pain syndrome after surgery. Anesthesiology 2004;101:1215-24.

9. Pak TJ, Martin GM, Magness JL, Kavanaugh GJ. Reflex sympathetic dystrophy: review of 140 cases. Minn Med 1970;53:507-12.

10. McFarlane RM, McGrouther DA. Complications and their management. In: Hueston, JT, and Tubiana, R, eds: Dupuytren's Disease. Edinburgh: Churchill Livingstone, 1985:377-82.

11. Zemel NP, Balcomb TV, Stark HH, et al. Dupuytren's disease in women: evaluation of long-term results after operation. J Hand Surg 1987;12:1012-6.

12. Tubiana R, Fahrer M, McCullough CJ. Recurrence and other complications in surgery of Dupuytren's contracture. Clin Plast Surg 1981;8:45-50.

13. Sennwald GR. Fasciectomy for treatment of Dupuytren's disease and early complications. J Hand Surg 1990;15:755-61.

14. Prosser R, Conolly WB. Complications following surgical treatment for Dupuytren's contracture. J Hand Ther 1996;9:344-8.

15. Viel EJ, Pelissier J, Eledjam JJ. Sympathetically maintained pain after surgery may be prevented by regional anesthesia. Anesthesiology 1994;81:265-6.

16. Reuben SS. Sympathetically maintained pain and the use of regional anesthesia. Anesthesiology 1994;81:1548.

17. Rocco AG. Sympathetically maintained pain may be rekindled by surgery under general anesthesia. Anesthesiology 1993;79: 865.

18. Reuben SS, Rosenthal EA, Steinberg RB. Surgery on the affected upper extremity of patients with a history of complex regional pain syndrome: a retrospective study of 100 patients. J Hand Surg 2000;25:1147-51.

19. Moore DC . Block of the stellate ganglion. In: Moore DC, ed. Regional block, 2nd ed. Springfield, IL: Charles C. Thomas, 1957:102-12.

20. Hogan QH, Taylor ML, Goldstein M, et al. Success rates in producing sympathetic block by paratracheal injection. Clin J Pain 1994;10:139-45.
21. Jahad AJ, Caroll D, Glynn CJ, McQuay HJ. Intravenous regional sympathetic blockade for pain relief in reflex sympathetic dystrophy: a systematic review and a randomized, double-blind crossover study. J Pain Symptom Manage 1995;10:13-20.

22. Kingery WS. A critical review of controlled clinical trials for peripheral neuropathic pain and complex regional pain syndromes. Pain 1997;73:123-39.

23. Perez RSGM, Kwakkel G, Zuurmond WWA, de Lange JJ. Treatment of reflex sympathetic dystrophy (CRPS Type 1): a research synthesis of 21 randomized clinical trials. J Pain Symptom Manage 2001;21:511-26.

24. Reuben SS, Steinberg RB, Klatt JL, Klatt ML. Intravenous regional anesthesia using lidocaine and clonidine. Anesthesiology 1999;91:654-8.

25. Reuben SS, Steinberg RB, Madabhushi L, Rosenthal E. Intravenous regional clonidine in the management of sympathetically maintained pain. Anesthesiology 1998;89:527-30.

26. Reuben SS, Rosenthal EA, Steinberg RB, Faruqi S. Surgery on the affected upper extremity of patients with a history of complex regional pain syndrome: the use of intravenous regional anesthesia with clonidine. J Clin Anesth 2004;16:517-22.

27. Kiowski W, Hulthen UI, Ritz R, Buhler FR. Prejunctional $\alpha 2-$ adrenoceptors and norepinephrine release in the forearm of normal humans. J Cardiovasc Pharmacol. 1985;7(suppl):S144-8.

28. Raja SN, Treede RD, Davis KD, Campbell JN. Systemic $\alpha$-adrenergic blockade with phentolamine: a diagnostic test for sympathetically maintained pain. Anesthesiology 1991;74: 691-8.

29. Ghostine SY, Comair YG, Turner DM, et al. Phenoxybenzamine in the treatment of causalgia. J Neurosurg 1984;60:1263-8.

30. Abram SE, Lightfoot RW. Treatment of long-standing causalgia with prazosin. Reg Anesth 1981;6:79-81.

31. McKain CW, Urban BJ, Goldner JL. The effects of intravenous regional guanethidine and reserpine: a controlled study. J Bone Joint Surg Am 1983;65:808-11.

32. Wallin G, Torebjörk E, Hallin RG. Preliminary observations on the pathophysiology of hyperalgesia in the causalgic pain syndrome. In: Zotterman Y, ed. Sensory functions of the skin of primates with special reference to man. Oxford: Pergamon, 1976:489-99.

33. Davis KD, Treede RD, Raja SN, et al. Topical application of clonidine relieves hyperalgesia in patients with sympathetically maintained pain. Pain 1991;47:309-17.

34. Gschwind C, Fricker R, Lacher G, Jung M. Does peri-operative guanethidine prevent reflex sympathetic dystrophy? J Hand Surgery 1995;20:773-5.

35. Hannington-Kiff JG. Intravenous regional sympathetic block with guanethidine. Lancet 1974;1:1019-20.

36. Hutchinson DT, McClinton MA. Upper extremity tourniquet tolerance. J Hand Surg 1993;18:206-10.

37. Crews JC, Denson DD, Hilgenhurst G, et al. Tourniquet pain: the response to the maintenance of tourniquet inflation on the upper extremity of volunteers. Reg Anesth 1991;16:314-7.

38. Lurie SD, Reuben SS, Gibson CS, et al. Effect of clonidine on upper extremity tourniquet pain in healthy volunteers. Reg Anesth Pain Med 2000;25:502-5.

39. Cousins MJ, Power I, Smith G. 1996 Labat Lecture: Pain: a persistent problem. Reg Anesth Pain Med 2000;25:6-21.

40. Cramer G, Young BM, Schwarzentraub P, et al. Preemptive analgesia in elective surgery in patients with complex regional pain syndrome: a case report. J Foot Ankle Surg 2000;39:387-91.

41. Ribbers GM, Geurts AC, Stam HJ, Mulder T. Pharmacologic treatment of complex regional pain syndrome I: a conceptual framework. Arch Phys Med Rehabil 2003;84:141-6.

42. Jänig W. The puzzle of "reflex sympathetic dystrophy": mechanisms, hypotheses, open questions. In: Stanton-Hicks, M, Jänig W, eds. Reflex sympathetic dystrophy: a reappraisal. Seattle: IASP Press, 1997:79-92. 